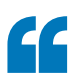

the prevalence

of arthritis

in 2015 was

estimated

at $36.8 \%$ in

the US adult

population

\title{
$\Rightarrow$ EPIDEMIOLOGY
}

Arthritis more common than expected

The prevalence of arthritis in the United States is much higher than previously estimated, particularly in younger adults, according to a new study published in Arthritis \& Rheumatology. "Our findings mean that arthritis should not be perceived as a condition that only requires attention in the elderly population," states S. Reza Jafarzadeh, corresponding author of the study.

In the United States, estimates of the prevalence of arthritis commonly rely on self-report surveys such as the National Health Interview Survey (NHIS). The national estimate for the prevalence of arthritis in 2015 relied on a single question from the NHIS regarding whether an individual had physician-diagnosed arthritis and did not take into account other arthritisrelated survey questions. To more accurately estimate the prevalence of arthritis, Jafarzadeh et al. developed surveillance criteria based on three NHIS questions relating to physician-diagnosed arthritis, chronic joint symptoms and the duration of these symptoms; to infer population parameters the researchers used statistical modelling. "Our analytic approach explicitly adjusts for the misclassification that is driven by the imperfect accuracy of the arthritis-related questions in the NHIS survey," explains Jafarzadeh. "This approach allows an estimation of the true prevalence of arthritis from aggregate-level data."

Using this approach, the prevalence of arthritis in 2015 was estimated at $36.8 \%$ in the US adult population (91.2 million adults of the 247.7 million projected total population), which was $68 \%$ higher than the previously reported 2015 national estimate of $22.7 \%$ (54.5 million adults). Furthermore, 30.6\% of younger adults (individuals aged 18-64, 61.1 million adults) had arthritis. Of the three surveillance criteria, the physician-diagnosed arthritis criterion had the lowest sensitivity in the younger adult group when stratified for age and sex, whereas the duration of symptoms criterion had the highest sensitivity.

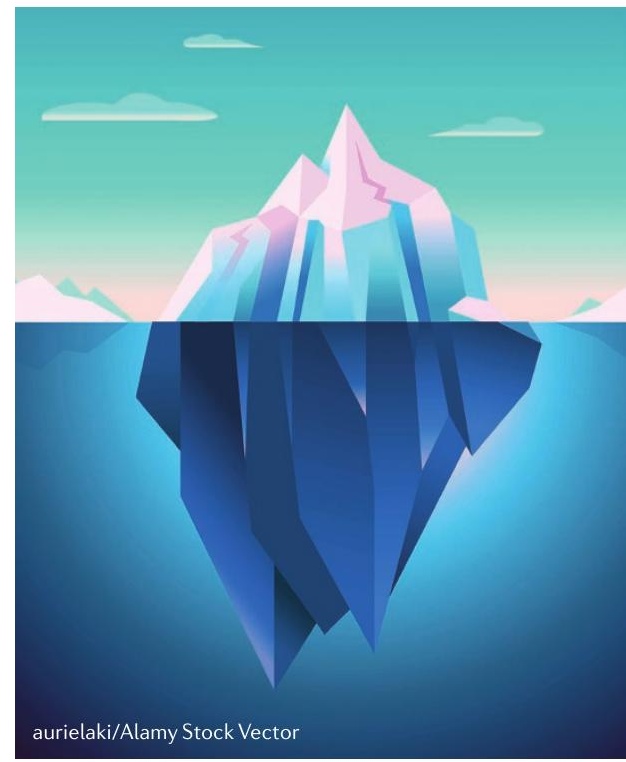

"Our study suggests that arthritis prevalence in the US adult population has been substantially underestimated, especially in adults below 65 years of age," remarks Jafarzadeh. "Future research could focus on the better monitoring of arthritis and on increasing arthritis awareness and prevention, which should improve the wellbeing of the population, especially for younger adults."

Jessica McHugh

ORIGINAL ARTICLE Jafarzadeh, S. R. \&

Felson, D. T. Updated estimates suggest a much higher prevalence of arthritis in US adults than previous ones. Arthritis Rheumatol. http://dx.doi. org/10.1002/art.40355 (2017) 